\title{
Comprehensive Dental Management of a Medically Compromised Individual under Anticoagulant Therapy - A Victim of Rabid Dog Bite
}

\author{
S. Karthik', Mukundan², R. Lakshmi Venkata Yamini ${ }^{3}$ \\ 1,2 Private Practioner, Mercy Multispecialty Dental Centre, Chennai, Tamil Nadu, India, ${ }^{3}$ Private Practioner, Kasthuri \\ Multispeciality Hospital, Chennai, Tamil Nadu, India
}

\begin{abstract}
In India, about 15 million people are bitten by animals, mostly dogs, every year and need post-exposure prophylaxis. Most animal bites in India (91.5\%) are by dogs, of which about $60 \%$ are strays and $40 \%$ pets. The incidence of animal bites is $17.4 / 1000$ population. Oral anticoagulants are usually prescribed for patients recovering from cardiovascular diseases, ischemic incidents such as myocardial infarction and angina pectoris followed by cerebrovascular accidents. Patients are usually placed under antiplatelet or oral anticoagulants increase the hemorrhage risk after oral surgical procedures. Here, we report a case of a patient on anticoagulants who, while seeking dental treatment, was bitten by a rabid street dog and shed light on the decisions faced by a dental professional while treating such patients.
\end{abstract}

Key words: Dental, anticoagulant, dog bite

\section{INTRODUCTION}

In India, about 15 million people are bitten by animals, mostly dogs, every year and need post-exposure prophylaxis. Most animal bites in India (91.5\%) are by dogs, of which about $60 \%$ are strays and $40 \%$ pets. The incidence of animal bites is $17.4 / 1000$ population. ${ }^{[1]}$

India has an estimated dog:man ratio of 1:36. The dogs fall into four broad categories: Pets (restricted and supervised); family dogs (partially restricted, wholly dependent); community dogs (unrestricted, partially dependent); and feral dogs (unrestricted, independent). ${ }^{[2]}$ Rabies is a hazardous viral condition that results when the treatment for a rabid dog bite is significantly delayed, because of the

\begin{tabular}{|c|l|}
\hline \multicolumn{2}{|c|}{ Access this article online } \\
\hline \multicolumn{1}{|c|}{ Publisher } & Website: \\
& Www.ijdms.in \\
& DOI: 10.30954/IJDMS.1.2020.11 \\
\hline
\end{tabular}

large number of bacteria. The oral animal bite wounds are generally contaminated, and their treatment is painful because of the risk of infection, especially in extensive injuries. ${ }^{[3]}$ Foundational to immediate wound care is appropriate hemostasis, antibiotic coverage, and rabies vaccination. Antibiotic therapy is indicated for infected bite wounds and fresh wounds considered at risk for infection. ${ }^{[4]}$ Oral anticoagulants are usually prescribed for patients recovering from cardiovascular diseases, ischemic incidents such as myocardial infarction and angina pectoris followed by cerebrovascular accidents. Patients are usually placed under antiplatelet or oral anticoagulants increase the hemorrhage risk after oral surgical procedures. Here, we report a case of a patient on anticoagulants who, while seeking dental treatment, was bitten by a rabid street dog and shed light on the decisions faced by a dental professional while treating such patients.

\section{CASE REPORT}

A healthy 50 -year-old male who was working as a pharmacist reported to the dental clinic with a chief complaint of food

\section{Address for Correspondence:}

Dr. S. Karthik, Consultant Dental Surgeon, Consultant Oral and Maxillofacial Pathologist, Mercy Multispecialty Dental Centre, 8/27, Parvathipuram $1^{\text {st }}$ Street, Thiruneermalai Main Road, Chennai - 600 044, Tamil Nadu, India. E-mail drkarthiks1981@gmail.com 
lodgment in his upper right back tooth region for the past 2 weeks. His medical history revealed that he had a history of coronary angiogram performed 2 years ago and had his coronary blockage dissolved by anticoagulant medication and has since been on maintenance drug regimen of clopidogrel and aspirin. He had diabetes, hypertensive was under medication (metformin and telmisartan, respectively) on the date of the visit. He had no other contributing medical history. On clinical examination, he had deposits of plaque and calculus around his tooth and Class-II decay in the mesio-occlusal part of 16. Hence, treatment was oral prophylaxis followed by caries excavation, restoration followed by prosthetic rehabilitation with porcelain fused met crown in relation to 14,15 , and 16 , respectively. The patient was advised to obtain a fitness certificate to undergo the treatment mentioned above procedure from a cardiologist and also his diabetologist.

The patient had returned with his fitness certificate; he was declared fit to undergo the procedure. He was placed under regular protocol followed by patients under anticoagulation medication was advised to stop taking his anticoagulant drugs 5 days prior and 5 days after the dental treatment. He was also placed under antibiotic prophylaxis with amoxicillin and clavulanic acid $(625 \mathrm{mg})$ to prevent any chances of infective endocarditis. The patient reported to the dental clinic after 5 days. He underwent oral prophylaxis, which included scaling and root planning followed by caries excavation in 16 and light cure composite restoration in 16. The prosthetic rehabilitation was scheduled for another appointment. The patient left satisfied. On the $2^{\text {nd }}$ day after the treatment, the patient bitten by a dog on his right forearm in the streets; he had called the dental office regarding the bite for which he was referred to a general physician's clinic. The patient then underwent routine protocol for dog bite wounds. The wound itself he sustained was not severe; there were no lacerations, just deep indentations of the dog's canine tooth followed by little bleeding that had clotted soon since the patient was not on any anticoagulant medication at the time of the incident. He then underwent vaccination for a rabies virus (Rabivax) for 14 days. After this, he was monitored regularly, and the wound had healed uneventfully. The prosthetic rehabilitation appointment was then postponed 6 months later. However, the treatment has been significantly pushed back due to the ongoing coronavirus disease-19 pandemic.

\section{DISCUSSION}

In India, about 15 million people are bitten by animals, mostly dogs, every year and need post-exposure prophylaxis. Most animal bites in India (91.5\%) are by dogs, of which about $60 \%$ are strays and $40 \%$ pets. The incidence of animal bites is 17.4 per 1000 population. ${ }^{[1]}$ Dog bite-related infections are polymicrobial, predominantly, Pasteurella and Bacteroides spp. infected bites presenting $<12 \mathrm{~h}$ after an injury are particularly likely to be infected with Pasteurella spp., whereas those presenting more than $24 \mathrm{~h}$ after the event are likely to be predominantly infected with staphylococci or anaerobes. The clinical approach in the UK to the management of dog bites is pragmatic; ${ }^{[5]}$ the NHS recommends co-amoxiclav as the first choice prophylaxis where indicated since it covers all the commonly expected organisms among the canine oral flora; ${ }^{[6]}$ in our patient, he had already been placed in antibiotic prophylaxis which covered the need for medication when the dog bite had occurred.

The oral anticoagulants are commonly prescribed for preventing complications such as thromboembolism, stroke in patients with prosthetic heart valves, congestive heart failures, and valvular defects. A complete history should always be attained along with the dosage of the anticoagulant medications taken by the patient. Patients on anticoagulant therapy should be delicately handled in a dental setup. Close collaboration with the patient's physician given utmost importance in these cases. In patients receiving long-term anticoagulant therapy, an international normalized ratio (INR) check $72 \mathrm{~h}$ before surgery is recommended. This allows sufficient time for dose modification if necessary to ensure a safe INR (2-4) on the day of dental surgery (including subgingival scaling). There is no need to check the INR for non-invasive dental procedures. ${ }^{[7]}$ It is necessary to carefully evaluate the bleeding risk of the planned treatment as well as the thrombotic risk of suppressing the anticoagulant or antiplatelet medication for each patient. ${ }^{[8]}$

The dentist must consult with the physician and verify that the patient's systemic status is acceptable for the minor surgical procedure. The purpose of the consultation is first, the confirmation of INR value within the therapeutic range for dental surgical procedures. Second, it is to let the physician know that the patient will be taken some kind of minor dental surgery. Third, it is to schedule the dental surgery appointment 2-3 days after the patient's routine INR check appointment, if possible.

However, in our case, the patient did not have any significant bleeding sustained by the dog bite apart from deep indentation, minor bleeding spots. Since he had discontinued oral anticoagulant, we could assume that his excessive bleeding was not seen due to increased platelets count due to stoppage of the oral anticoagulant medication, the antibiotic is taken by the patient to prevent any probability of infective endocarditis also covered for the antibiotic coverage that had to be given due to the dog bite. After deeming the patient fit, he was treated to his problems accordingly.

\section{CONCLUSION}

Regardless of the procedure to be carried out, the overall medical condition of the patient should be given more 
emphasis. It is advisable to take into account all the local hemostatic measures known. Concerning conventional antiplatelet drugs, the most common tendency is not to withdraw the drug provided as long as post-operative bleeding is controlled. However, if proper history is not available, it is better to stop the antiplatelet medication until the dental treatment is completed to avoid unnecessary hemorrhage. A dental surgeon should be capable of treating a dog bite and should possess the courage to handle a delicate situation. The dentist should work in close collaboration with the other respective physicians for better treatment plans and the welfare of the patient.

\section{REFERENCES}

1. Sudarshan MK. Assessing burden of rabies in India. WHO sponsored national multi-centric rabies survey. Assoc Prev Control Rabies India J 2004;6:44-5.

2. Chaudhuri S. Rabies Prevention and Dog Population Management.
India's Official Dog Control Policy in Context of WHO Guidelines, Ecollage; 2005.

3. Wolff KD. Management of animal bite injuries of the face: Experience with 94 patients. J Oral Maxillofac Surg 1998;56:838-43.

4. Stefanopoulos PK, Tarantzopoulou AD. Facial bite wounds: Management update. Int J Oral Maxillofac Surg 2005;34:464-72.

5. Morgan M, Palmer J. Dog bites. BMJ 2007;334:413-7.

6. Management of Animal Bites. Prodigy Guidance. Available from: http://www.prodigy.nhs.uk/qrg/bites_animal.pdfwww.prodigy. nhs.uk/qrg/bites_animal.pdf. [Last accessed on 2020 May 01].

7. Kahri J, Rapola J. Cardiovascular disorders in dental practice. Nor Tannlaegeforen Tid 2005;115:84-90.

8. Perry DJ, Noakes TJ, Helliwell PS, British Dental Society. Guidelines for the management of patients on oral anticoagulants requiring dental surgery. Br Dent J 2007;203:389-93.

How to cite this article: Karthik S, Mukundan, Yamini RLV. Comprehensive Dental Management of a Medically Compromised Individual under Anticoagulant Therapy - A Victim of Rabid Dog Bite. Int J Dent Med Spec 2020;7(1):46-48.

Source of Support: None; Conflicts of Interest: None 